\title{
3D Non-rigid Registration Using Surface and Local Salient Features for Transrectal Ultrasound Image-guided Prostate Biopsy
}

\author{
Xiaofeng Yang ${ }^{1}$, Hamed Akbari ${ }^{1}$, Luma Halig ${ }^{1}$, Baowei Fei ${ }^{1,2^{*}}$ \\ Departments of Radiology ${ }^{1}$ and Biomedical Engineering ${ }^{2}$, Emory University \\ * Corresponding Author: bfei@emory.edu
}

\begin{abstract}
We present a 3D non-rigid registration algorithm for the potential use in combining PET/CT and transrectal ultrasound (TRUS) images for targeted prostate biopsy. Our registration is a hybrid approach that simultaneously optimizes the similarities from point-based registration and volume matching methods. The 3D registration is obtained by minimizing the distances of corresponding points at the surface and within the prostate and by maximizing the overlap ratio of the bladder neck on both images. The hybrid approach not only capture deformation at the prostate surface and internal landmarks but also the deformation at the bladder neck regions. The registration uses a soft assignment and deterministic annealing process. The correspondences are iteratively established in a fuzzy-to-deterministic approach. B-splines are used to generate a smooth non-rigid spatial transformation. In this study, we tested our registration with pre- and postbiopsy TRUS images of the same patients. Registration accuracy is evaluated using manual defined anatomic landmarks, i.e. calcification. The root-mean-squared (RMS) of the difference image between the reference and floating images was decreased by $62.6 \pm 9.1 \%$ after registration. The mean target registration error (TRE) was $0.88 \pm 0.16 \mathrm{~mm}$, i.e. less than 3 voxels with a voxel size of $0.38 \times 0.38 \times 0.38 \mathrm{~mm}^{3}$ for all five patients. The experimental results demonstrate the robustness and accuracy of the $3 \mathrm{D}$ non-rigid registration algorithm.
\end{abstract}

Keywords: Tranrectal ultrasound (TRUS), non-rigid registration, PET/CT, image-guided prostate biopsy, molecular imaging, image registration, prostate cancer, targeted biopsy of the prostate

\section{INTRODUCTION}

Prostate cancer is the second leading cause of cancer death in men in the United States [1]. Transrectal ultrasound (TRUS)-guided biopsy is the clinical standard for definitive diagnosis of prostate cancer. While 2D TRUS-guided biopsy is routinely performed, however, 2D TRUS images do not provide 3D location of the biopsy sample. Consequently, the physician must mentally estimate the 3D location of the biopsy needle based on limited 2D information, thus leading to suboptimal biopsy targeting. As combined PET/CT can offer metabolic, functional, and anatomic information, the metabolic images from PET would be able to be used to direct targeted biopsy of the prostate by registering PET/CT images with TRUS images.

Image registration has become a useful tool for various applications such as longitudinal studies [2], population-based disease studies [3], image information fusion [4,5,6], and image guided intervention [7,8]. Multimodality image registration can help to provide a comprehensive understanding of anatomic or pathologic structure by integrating information gained from different imaging modalities. Non-rigid registration $[9,10]$ is the building block for a variety of medical image analysis tasks, such as multi-modality information fusion, atlas-based image segmentation and computational anatomy. Existing non-rigid registration methods can be generally classified into two main categories: voxel-wise/intensity-based methods [11] and landmark/feature-based methods [12]. Feature-based registration methods use sparse features extracted from images, such as points [13], curves, and surface patches. Registration is find their correspondences and compute an optimal transformation. The key for feature-based methods is to find true correspondences between two feature sets. Most of them use Euclidean distance-based geometric features to solve correspondences, for example, the iterative closest point algorithm [12,14,15], the soft-assign method [16,17], shape

Medical Imaging 2011: Visualization, Image-Guided Procedures, and Modeling,

edited by Kenneth H. Wong, David R. Holmes III, Proc. of SPIE Vol. 7964, 79642V

(C) 2011 SPIE $\cdot$ CCC code: $1605-7422 / 11 / \$ 18 \cdot$ doi: $10.1117 / 12.878153$

Proc. of SPIE Vol. 7964 79642V-1 
context-based methods [18], and kernel correlation-based methods [19,20]. Some of them use intensity-based local similarity measures, such as cross correlation, mutual information to determine correspondences [21,22]. Some hybrid methods integrate geometric features and intensity based local similarity measures for computing correspondences $[23,24]$. In this paper, we combine point-based registration and volume matching method in order to improve accuracy and robust of prostate registration.

\section{METHODS}

Our non-rigid registration method includes three terms: (1) surface landmark matching, (2) internal landmark matching, (3) volume overlap matching. The schematic diagram of our registration method is shown in Figure 1, where $x_{i}^{C T}$ and $y_{i}^{U S}$ are surface landmarks of the prostate from the segmented CT and TRUS images, respectively, $u_{k}^{C T}$ and $v_{l}^{U S}$ are internal landmarks e.g. urethra and calcification within the prostate on the CT and TRUS images, respectively. $B_{C T}$ and $B_{U S}$ represent the bladder neck region on the CT and TRUS images, respectively.

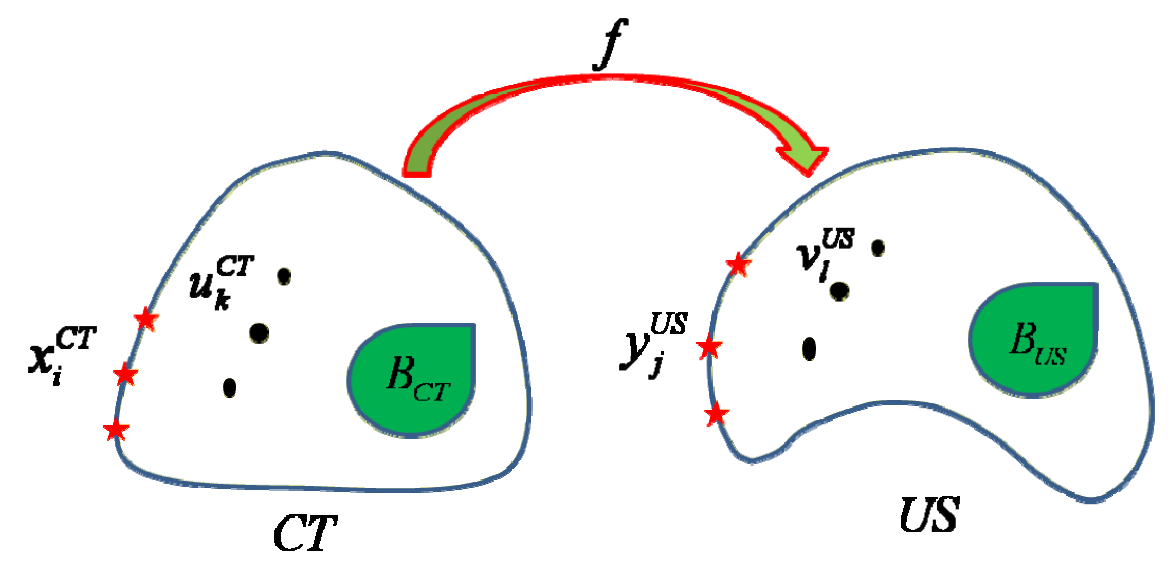

Figure 1. The schematic diagram of our registration method.

Inspired by $[25,26,27]$, we design an overall similarity function to integrate the similarities between same type of landmarks and add smoothness constraints on the estimated transformation between segmented CT and TRUS images. The transformation between CT and TRUS images are represented by a general function, which can be modeled by various function basis. In our study, we choose B-splines as the transformation basis. The similarity function is written as:

where

$$
\begin{gathered}
\min _{f} E(f)=\alpha E_{S S}\left(f\left\{x_{i}^{C T}, y_{j}^{U S}\right\}\right)+\beta E_{I S}\left(f\left\{u_{k}^{C T}, v_{l}^{U S}\right\}\right)+\gamma E_{V M}\left(f\left\{B_{C T}, B_{U S}\right\}\right)+\lambda E_{S}(f) \\
E_{S S}(f)=\sum_{i=1}^{I} \sum_{j=1}^{J} p_{i j}\left(\left\|y_{j}^{U S}-f\left(x_{i}^{C T}\right)\right\|^{2}\right)+\delta \sum_{i=1}^{I} \sum_{j=1}^{J} p_{i j} \log \left(p_{i j}\right)-\xi \sum_{i=1}^{I} \sum_{j=1}^{J} p_{i j} \\
E_{I S}(f)=\sum_{k=1}^{K} \sum_{l=1}^{L} q_{k l}\left\|v_{l}^{U S}-f\left(u_{k}^{C T}\right)\right\|^{2}+\tau \sum_{k=1}^{K} \sum_{l=1}^{L} q_{k l} \log \left(q_{k l}\right)-\eta \sum_{k=1}^{K} \sum_{l=1}^{L} q_{k l} \\
E_{V M}(f)=1-\frac{2^{*}\left(f\left(B_{C T}\right) \bigcap B_{U S}\right)}{\left|f\left(B_{C T}\right)\right|+\left|B_{U S}\right|}
\end{gathered}
$$




$$
E_{S}(f)=\left\|\iiint_{(x, y, z) \in \Omega_{M}}\left(\frac{\partial^{2} f}{\partial x^{2}}+\frac{\partial^{2} f}{\partial y^{2}}+\frac{\partial^{2} f}{\partial z^{2}}\right)^{2} d x d y d z\right\|^{2}
$$

$\alpha, \beta, \gamma$, and $\lambda$ are the weights for each energy term. $E_{S S}$ is the similarity for surface landmarks, and $E_{I S}$ is the similarity for internal landmarks. $E_{V M}$ is the energy term for the bladder-neck volume matching; and $E_{S}$ is the smoothness constraint term. $\delta$ and $\tau$ are called the temperature parameter and its weighted term is an entropy term comes from the deterministic annealing technique [28]. $\xi$ and $\eta$ are the weight for the outlier rejection term. Matrixes $p_{i j}$ and $q_{k l}$ are the fuzzy correspondence matrixes [25]. $f$ denotes the transformation between CT and TRUS images [29].

The overall similarity function can be minimized by an alternating optimization algorithm that successively updates the correspondences matrixes $p_{i j}$ and $q_{k l}$, and the transformation function $f$. First, with the fixed transformation $f$, the correspondence matrixes between landmarks are updated by minimizing $E(f)$. The updated correspondence matrixes are then treated as the temporary correspondences between landmarks. Second, with the fixed temporary correspondence matrixes $p_{i j}$ and $q_{k l}$, the transformation function $f$ is updated. The two steps are alternatively repeated until there are no updates of the correspondence matrixes.

In order to evaluate the accuracy and robustness of the registration method, we calculate the root mean squared $(R M S)$ difference between the original and registered images [27]. Second we used target registration errors (TRE) [30] to evaluate the registration accuracy. We used eight visible calcifications that were identified on both images as the anatomic landmarks. 


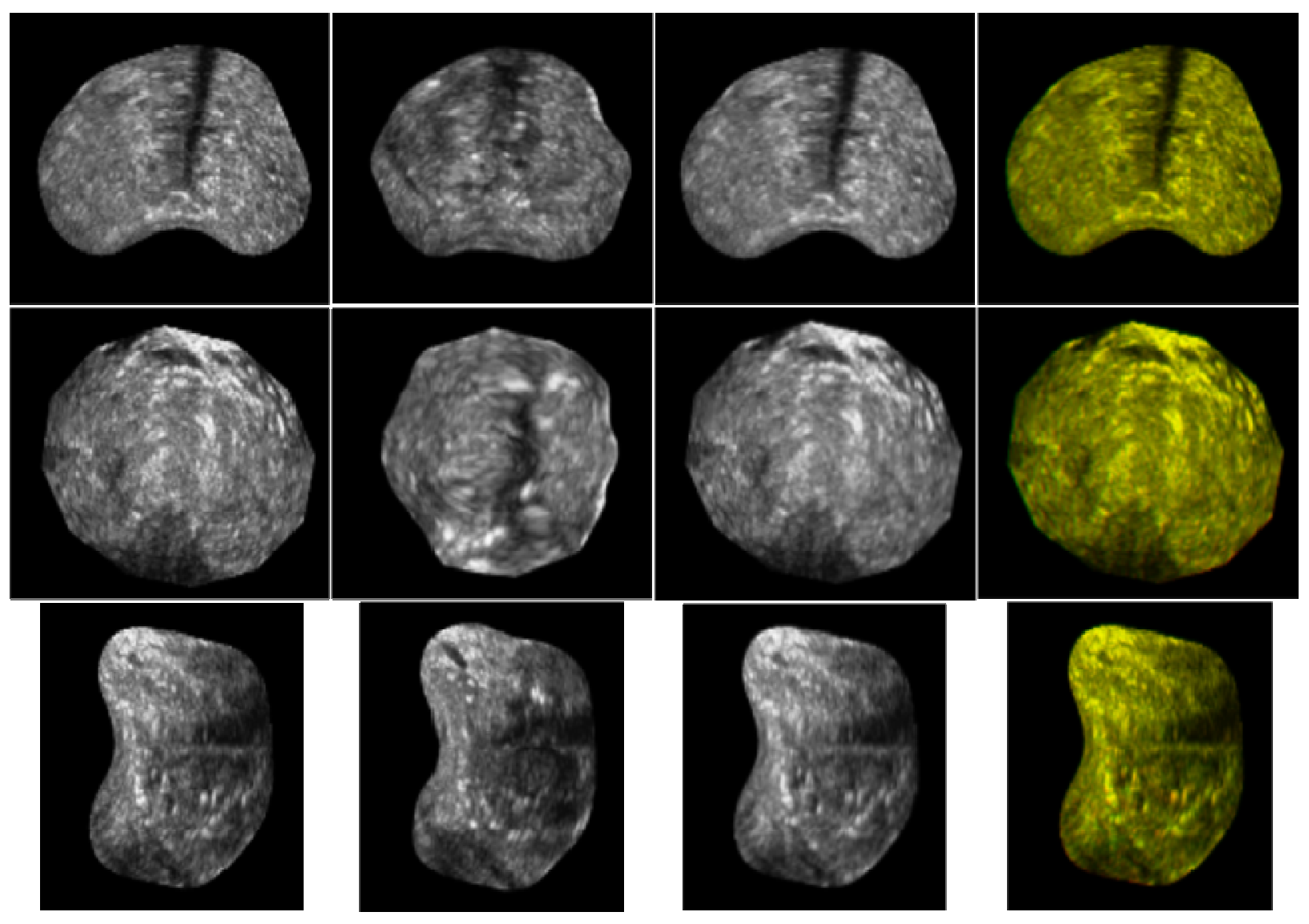

Figure.2 Registration between original (left column) and simulated images (second column) with deformation. The third column is the registered images after deformable registration. The fourth column is the fusion between the original and registered images.

\section{RESULTS}

Our registration method has been evaluated using simulated and real TRUS images. We used the original TRUS images as reference images, deformed the images to simulate the floating images, and then registered the deformed images to the original images. Figure 2 illustrates the visual assessment of the registration results in three directions. There is significant difference between original and deformed images before registration. However, after our registration the registered images are matched to the original images. It demonstrates the feasibility of the registration method.

Our non-rigid registration method was also evaluated using five sets of pre- and post-biopsy TRUS data of the same patients. The size of TRUS data is $244 \times 244 \times 175$ voxels and the spatial resolution is $0.38 \times 0.38 \times 0.39 \mathrm{~mm}^{3}$. We used prebiopsy images as the reference images and registered the post-biopsy images of the same patient. Figure 3 illustrates the visual assessment of the registration results of one patient. For five sets of patient data, the RMS and TRE are shown in Figure 4 and 5 . The RMS difference was decreased by $62.6 \pm 9.1 \%$ after registration. The TRE was $0.88 \pm 0.16 \mathrm{~mm}$ and the maximum TRE is $1.08 \pm 0.21 \mathrm{~mm}$. 

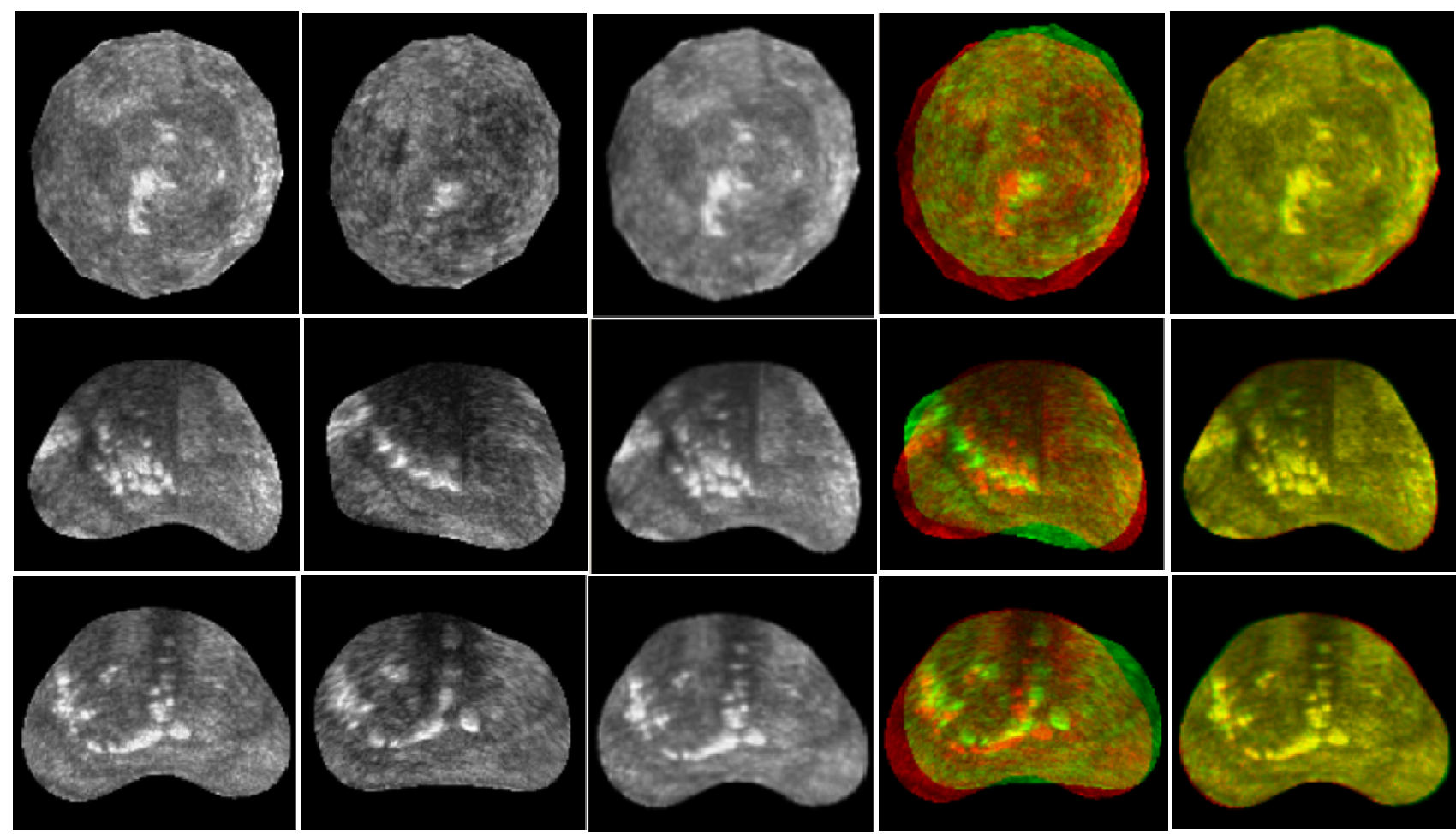

Figure.3 Registration results of pre-biopsy (left column) and post-biopsy TRUS images ( $2^{\text {nd }}$ column) of the same patient. $3^{\text {rd }}$ column: Registered post-biopsy images. The fusion of pre- and post-biopsy images before registration $\left(4^{\text {th }}\right.$ column) and after registration $\left(5^{\text {th }}\right.$ column) shows the improvement of the matching between the two images.

\section{CONCLUSION}

We develop and evaluate a 3D non-rigid registration method that combines point-based registration and volume overlap matching methods. The registration method can be used to register 3D TRUS images acquired at different time points and can be used for potential use in TRUS-guided prostate re-biopsy. We have tested 3D non-rigid registration algorithm for pre- and post-biopsy TRUS images. Our next step is to apply this method to CT and TRUS images and then incorporate PET/CT images into TRUS-guarded prostate biopsy in patients. 


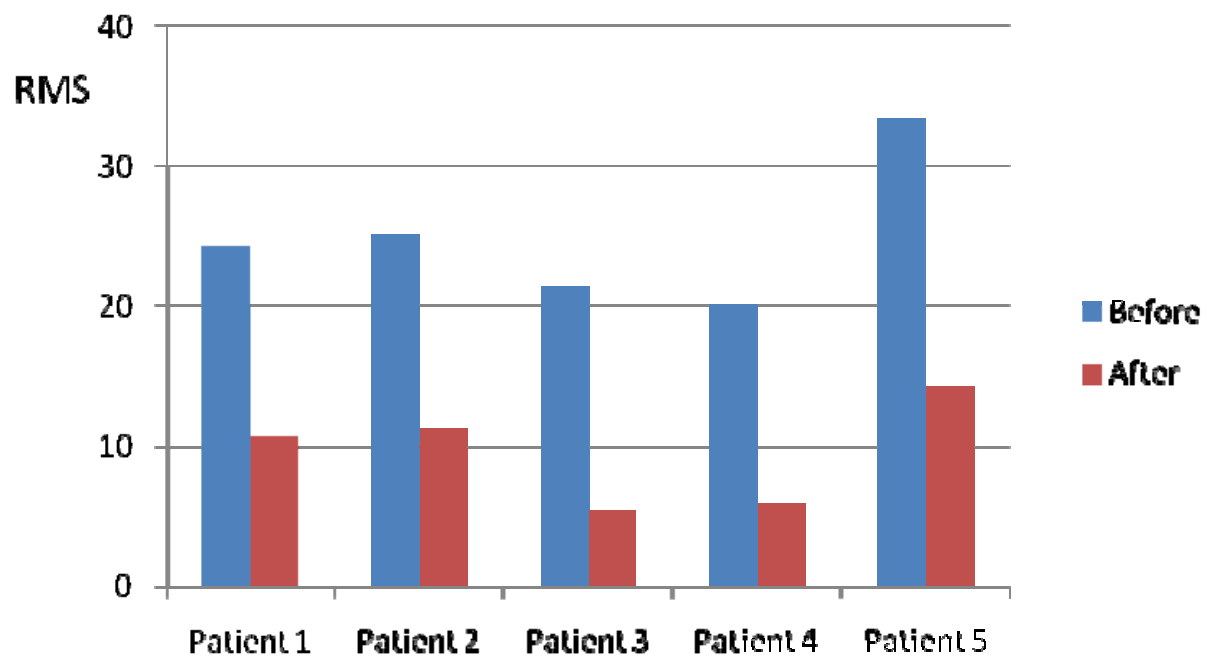

Figure.4. The root-mean-squared (RMS) of the intensity of the difference image between the reference and floating images before and after registration.

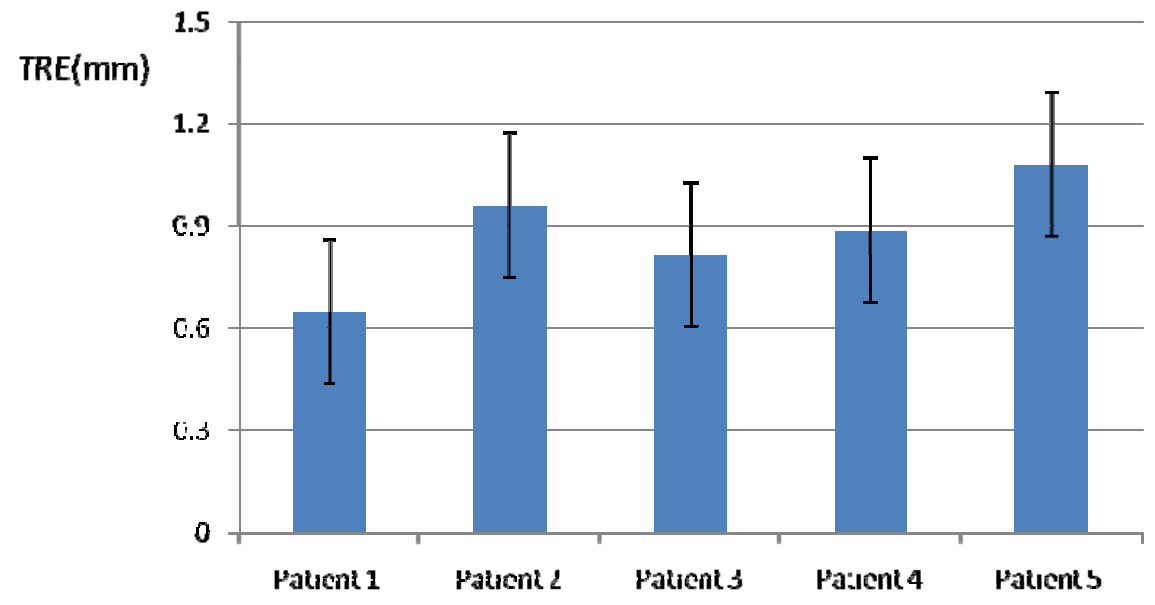

Figure.5. Target registration errors (TRE) between pre-biopsy and post-biopsy TRUS image. For each patient eight anatomic landmarks were selected to calculate the TRE. Note the voxel size of the TRUS images is $0.38 \times 0.38 \times 0.38$ $\mathrm{mm}^{3}$. Error bars are standard deviations.

\section{ACKNOWLEGEMENT}

This research is supported in part by NIH grant R01CA156775 (PI: Fei), Coulter Translational Research Grant (PIs: Fei and $\mathrm{Hu}$ ), Georgia Cancer Coalition Distinguished Clinicians and Scientists Award (PI: Fei), Emory Molecular and Translational Imaging Center (NIH P50CA128301), and Atlanta Clinical and Translational Science Institute (ACTSI) that is supported by the PHS Grant UL1 RR025008 from the Clinical and Translational Science Award program. We thank Dr. Aaron Fenster at Robart Research Institute of The University of Western Ontario for providing the ultrasound images. 


\section{Reference}

[1] Jemal A, Siegel R, Xu J, Ward E. "Cancer statistics 2010," CA: a cancer journal for clinicians, 60, 277-300 (2010).

[2] Thompson,P.M., Mega,M.S., Woods,R.P., Zoumalan,C.I., Lindshield,C.J., Blanton,R.E., Moussai,J., Holmes,C.J., Cummings,J.L., Toga,A.W., "Cortical change in Alzheimer's disease detected with a diseasespecific population-based brain atlas," Cereb. Cortex. 11(1):1-16 (2001).

[3] Fan,Y., Shen,D., and Davatzikos,C., "Classification of Structural Images via High-Dimensional Image Warping, Robust Feature Extraction, and SVM," Med Image Comput Comput Assist Interv. 8(Pt 1):1-8 (2005).

[4] Rouet,J.M., Jacq,J.J., and Roux,C., "Genetic algorithms for a robust 3-D MR-CT registration," IEEE Trans Inf. Technol. Biomed. 4(2):126-36 (2000).

[5] Fei,B., Lee,Z., Duerk,J.L., Lewin,J., Sodee,D., and Wilson,D.L., "Registration and Fusion of SPECT, High Resolution MRI, and interventional MRI for Thermal Ablation of the Prostate Cancer," IEEE Transactions on Nuclear Science.51(1), 177-183 (2004).

[6] Fei,B., Lee,Z., Boll DT, Duerk JL, LewinJS, and ilson,D.L., "Image Registration and Fusion for Interventional MRI Guided Thermal Ablation of the Prostate Cancer," The Sixth Annual International Conference on Medical Imaging Computing \& Computer Assisted Intervention (MICCAI 2003). Lecture Notes in Computer Science (LNCS), 2879, 364-372 (2003).

[7] Hill,D.L., Hawkes,D.J., Gleeson,M.J., Cox,T.C., Strong,A.J., Wong,W.L., Ruff,C.F., Kitchen,N.D., Thomas,D.G., and Sofat,A., "Accurate frameless registration of MR and CT images of the head: applications in planning surgery and radiation therapy," Radiology.191, 447-454 (1994).

[8] Fei,B., Lee,Z., Duerk JL, and Wilson,D.L., "Image Registration for Interventional MRI Guided Procedures: Similarity Measurements, Interpolation Methods, and Applications to the Prostate," Proceedings of The Second International Workshop on Biomedical Image Registration, Lecture Notes in Computer Science (LNCS). 2717, 321-329 (2003).

[9] Fei,B., Wang,H., Muzic,R.F., Jr., Flask,C., Wilson,D.L., Duerk,J.L., Feyes,D.K., and Oleinick,N.L., "Deformable and rigid registration of MRI and microPET images for photodynamic therapy of cancer in mice," Med. Phys. 33, 753-760 (2006).

[10] Fei,B., Duerk,J.L., Sodee,D.B., and Wilson,D.L., "Semiautomatic nonrigid registration for the prostate and pelvic MR volumes," Acad. Radiol. 12, 815-824 (2005).

[11] Ou,Y. and Davatzikos,C., "DRAMMS: deformable registration via attribute matching and mutual-saliency weighting," Inf. Process Med Imaging. 21, 50-62 (2009).

[12] B.Zitova and J.Flusser, "Image registration methods: a survey," Image Vis. Comput.21(11), 977-1000 (2003).

[13] Bogie,K., Wang,X., Fei,B., and Sun,J., "New technique for real-time interface pressure analysis: getting more out of large image data sets," J Rehabil. Res. Dev. 45, 523-35 (2008).

[14] Z.Zhang, "Iterative point matching for registration of freeform curves and surfaces," Int. J. Comput. Vis.13(2), 119-152 (1992).

[15] Besl,P.J. and McKay,H.D., "A method for registration of 3-D shapes," Pattern Analysis and Machine Intelligence, IEEE Transactions on. 14, 239-256 (1992).

[16] Chui,H. and Rangarajan,A., "A new point matching algorithm for non-rigid registration," Computer Vision and Image Understanding.89(2-3), 114-141 (2002).

[17] A.Rangarajan, H.Chui, and F.Bookstein, "The softassign procrustes matching algorithm," In Proc. IPMI. 29-42 (1997).

[18] Belongie,S., Malik,J., and Puzicha,J., "Shape matching and object recognition using shape contexts," Pattern Analysis and Machine Intelligence, IEEE Transactions on.24, 509-522 (2002).

[19] Y.Tsin and T.Kanade, "A correlation-based approach to robust point set registration," In Proc. ECCV. 558-569 (2004).

[20] B.Jian and B.C.Vemuri, "A robust algorithm for point set registration using mixture of Gaussians," In Proc. ICCV. 1246-1251 (2005).

[21] Guimond,A., Roche,A., Ayache,N., and Meunier,J., "Three-dimensional multimodal brain warping using the demons algorithm and adaptive intensity corrections," IEEE Trans Med Imaging.20(1), 58-69 (2001).

[22] Cachier,P., Bardinet,E., Dormont,D., Pennec,X., and Ayache,N., "Iconic feature based nonrigid registration: the PASHA algorithm," Computer Vision and Image Understanding.89(2-3), 272-298 (2002). 
[23] P.Cachier, J.-F.Mangin, X.Pennec, D.Riviere, D.Papadopoulos-Orfanos, J.Regis, and N.Ayache, "Multisubject non-rigid registration of brain MRI using intensity and geometric features," MICCAI2001. 734-742 (2001).

[24] D.Shen and C.Davatzikos, "HAMMER: hierarchical attribute matching mechanism for elastic registration," IEEE Trans Med Imaging.21(11), 1421-1439 (2002).

[25] Chui,H. and Rangarajan,A., "A new point matching algorithm for non-rigid registration," Computer Vision and Image Understanding.89(2-3), 114-141 (2002).

[26] Zhan,Y., Ou,Y., Feldman,M., Tomaszeweski,J., Davatzikos,C., and Shen,D., "Registering histologic and MR images of prostate for image-based cancer detection," Acad. Radiol. .14, 1367-1381 (2007).

[27] Yang,J., Blum,R.S., Williams,J.P., Sun,Y., and Xu,C., "Non-rigid Image Registration Using Geometric Features and Local Salient Region Features," CVPR, 1825-832 (2006).

[28] Yuille,A.L. and Kosowsky,J.J., "Statistical Physics Algorithms That Converge," Neural Computation.6(3), 341356 (1994).

[29] Wang,H. and Fei,B., "A Robust B-Splines-Based Point Match Method for Non-Rigid Surface Registration," The 2nd International Conference on Bioinformatics and Biomedical Engineering (ICBBE2008), 2353-2356 (2008).

[30] Karnik,V.V., Fenster,A., Bax,J., Cool,D.W., Gardi,L., Gyacskov,I., Romagnoli,C., and Ward,A.D., "Assessment of image registration accuracy in three-dimensional transrectal ultrasound guided prostate biopsy," Med. Phys.37(2), 802-813 (2010). 
Yang $\mathrm{X}$, Akbari H, Halig L, Fei B. 3D non-rigid registration using surface and local salient features for transrectal ultrasound image-guided prostate biopsy. SPIE Medical Imaging: Visualization, Image-Guided Procedures, and Modeling, Edited by Kenneth H. Wong; David R. Holmes III, Editors, Proceedings of SPIE 2011;7964:79642V-1 8

Copyright 2011 Society of Photo-Optical Instrumentation Engineers (SPIE). One print or electronic copy may be made for personal use only. Systematic reproduction and distribution, duplication of any material in this paper for a fee or for commercial purposes, or modification of the content of the paper are prohibited.

http://dx.doi.org/10.1117/12.878153 